\title{
Translation efficiency of heterologous proteins is significantly affected by the genetic context of RBS sequences in engineered cyanobacterium Synechocystis sp. PCC 6803
}

\author{
Kati Thiel, Edita Mulaku, Hariharan Dandapani, Csaba Nagy, Eva-Mari Aro and Pauli Kallio*
}

\begin{abstract}
Background: Photosynthetic cyanobacteria have been studied as potential host organisms for direct solar-driven production of different carbon-based chemicals from $\mathrm{CO}_{2}$ and water, as part of the development of sustainable future biotechnological applications. The engineering approaches, however, are still limited by the lack of comprehensive information on most optimal expression strategies and validated species-specific genetic elements which are essential for increasing the intricacy, predictability and efficiency of the systems. This study focused on the systematic evaluation of the key translational control elements, ribosome binding sites (RBS), in the cyanobacterial host Synechocystis sp. PCC 6803, with the objective of expanding the palette of tools for more rigorous engineering approaches.
\end{abstract}

Results: An expression system was established for the comparison of 13 selected RBS sequences in Synechocystis, using several alternative reporter proteins (SYFP2, codon-optimized GFPmut3 and ethylene forming enzyme) as quantitative indicators of the relative translation efficiencies. The set-up was shown to yield highly reproducible expression patterns in independent analytical series with low variation between biological replicates, thus allowing statistical comparison of the activities of the different RBSs in vivo. While the RBSs covered a relatively broad overall expression level range, the downstream gene sequence was demonstrated in a rigorous manner to have a clear impact on the resulting translational profiles. This was expected to reflect interfering sequence-specific mRNA-level interaction between the RBS and the coding region, yet correlation between potential secondary structure formation and observed translation levels could not be resolved with existing in silico prediction tools.

Conclusions: The study expands our current understanding on the potential and limitations associated with the regulation of protein expression at translational level in engineered cyanobacteria. The acquired information can be used for selecting appropriate RBSs for optimizing over-expression constructs or multicistronic pathways in Synechocystis, while underlining the complications in predicting the activity due to gene-specific interactions which may reduce the translational efficiency for a given RBS-gene combination. Ultimately, the findings emphasize the need for additional characterized insulator sequence elements to decouple the interaction between the RBS and the coding region for future engineering approaches.

Keywords: Ribosome binding site, Translational efficiency, Genetic context, Synechocystis sp. PCC 6803

*Correspondence: pataka@utu.fi

Molecular Plant Biology, Department of Biochemistry, University of Turku, 20014 Turku, Finland 


\section{Background}

In response to increasing environmental concerns and exponentially growing demand for consumer products, there is an urgent global need to find sustainable alternatives for different carbon-based chemicals which are currently derived from non-renewable sources. As part of this development, photosynthetic cyanobacteria have been studied as potential next-generation biotechnological host organisms for the production of desired chemicals directly from atmospheric $\mathrm{CO}_{2}$ and water, using solar radiation as energy $[1,2]$. Although the proof of concept has been established for the technology, there are still various biological and technical shortcomings which critically restrict us from harnessing the photosynthetic capacity to a sufficient degree for commercial applications. In particular, the efficiency of converting the light energy into the target products is currently inadequate, and further development calls for more flexible strategies to enhance preparative throughput and expand chemical diversity beyond the conventional engineering approaches in cyanobacterial research. In order to overcome the constraints, systematic synthetic biology approaches relying on validated genetic control elements, modular assembly systems and optimized expression strategies are currently being evaluated in cyanobacterial hosts such as Synechocystis sp. PCC 6803 (Synechocystis from here on).

One of the typical challenges in metabolic engineering is to have precise control over the expression of the introduced genes in a predictable manner. Besides promoters which typically serve as the master switches in regulating expression at the transcription phase, ribosome binding site (RBS) sequences play a key role in determining the output by controlling the translation efficiency of individual ORFs. In addition to maximizing the expression level of a specific target protein, RBSs can be used for modulating the relative translation efficiencies of individual proteins in polycistronic pathways at a broad dynamic range [3]. Importantly, this provides the means for optimizing the performance of engineered pathways with multiple heterologous genes, in which the metabolic flux through the subsequent enzyme-catalyzed biosynthetic steps need to be balanced in order to function properly in context with the surrounding cellular metabolism [3]. However, unlike for established model organisms such as $E$. coli for which structure-function relationships in genetic regulation have already been extensively studied, the corresponding information on the function of RBSs in cyanobacteria is still rather limited to allow complex rational engineering.

Several studies have previously addressed the function and relative activities of RBSs in Synechocystis (Table 1) [4-7], but in many cases the RBSs and promoter sequences are either discussed together without clear distinction, or direct assessment of factors affecting the translational efficiency is difficult due to variation in context or experimental set-up between the publications. Although the nucleotide sequences around the RBS region are known to potentially impact the level of translation $[6,8,9]$, the phenomenon has not been systematically evaluated in cyanobacteria, and there is no clear consensus of the frequency or extent of the effect in engineered cyanobacterial systems. With the realization that the function of RBSs can be clearly species-specific, as observed for example between $E$. coli and Synechocystis $[4,10,11]$, such information could help to identify the most critical points in the design of new context-independent expression strategies. Currently, even though prominent in silico prediction tools have been developed and optimized to evaluate the function of RBS sequences [12-14], the correlation with the observed activities in cyanobacteria appear to be poor $[15,16]$, and should be used with caution if direct experimental validation is not available.

RBS is a short (typically less than $20 \mathrm{bp}$ ) nucleotide sequence at the $5^{\prime}$ UTR of mRNA, which guides the ribosome binding in the correct orientation in respect to the start codon, and thus allows translation to begin. As the initiation phase is the key rate-limiting step in translation, RBS constitutes an important control element in determining the accuracy and overall efficiency in protein expression. RBSs typically contain a specific guanidinerich core region called Shine-Dalgarno (SD) sequence, located about 5-13 bp upstream from the initiation codon. The consensus sequence for SD is $5^{\prime}$-GGAGG- $3^{\prime}$ $[4,11,17]$ which is recognized and bound by the complementary $3^{\prime}$ anti-SD sequence of the $16 \mathrm{~S}$ rRNA of the $30 \mathrm{~S}$ ribosomal subunit, resulting in the translation initiation complex formation. The efficacy of the primary interaction between the RBS and the ribosome depends on the (i) degree of complementarity between the SD core sequence and the $16 \mathrm{~S}$ rRNA, (ii) the distance of the SD from the translation initiation codon, and (iii) the surrounding nucleotide sequences, which may form secondary structures that interfere with the binding $[4,11,17]$. Despite high level of conservation in the SD sequences between prokaryotes, most RBSs show variations in the region. For example, while E. coli and Synechocystis share the same core anti-SD sequence corresponding to $5^{\prime}$-GGAGG-3', the respective percentage of genes carrying this sequence in the two organisms is 57 and $26 \%$, respectively. In addition, the optimal aligned spacing between the start codon and the SD core sequence has been reported to be 7-9 bp for E. coli but 9-11 bp for Synechocystis [11]. 
Table 1 RBSs subjected to in vivo characterization or comparative analysis in Synechocystis sp. PCC 6803 as reported in literature

\begin{tabular}{|c|c|c|c|}
\hline Name and sequence & Organism/source & Reporter & References \\
\hline $\begin{array}{l}\text { BBa_B0029 } \\
\text { ATTCACACAGGAAACCTACTAGATG } \\
\text { NTTCACACAGGAAACCTCTAGAATG }\end{array}$ & BioBrick & $\begin{array}{l}\text { mTagBFP } \\
\text { EYFP }\end{array}$ & $\begin{array}{l}{[6]} \\
{[6]}\end{array}$ \\
\hline $\begin{array}{l}\text { BBa_B0030 } \\
\left.(\mathrm{N})_{n} \text { ATTAAAGAGGAGAAA(N) }\right)_{n} \text { ATG } \\
\text { AATTAAAGAGGAGAAATACTAGATG } \\
\text { NATTAAAGAGGAGAATCTAGAATG }\end{array}$ & BioBrick & $\begin{array}{l}\text { GFPmut3b } \\
\text { mTagBFP } \\
\text { EYFP }\end{array}$ & $\begin{array}{l}{[4]} \\
{[6]} \\
{[6]}\end{array}$ \\
\hline $\begin{array}{l}\text { BBa_B0031 } \\
\text { GATCACACAGGAAACCTACTAGATG } \\
\text { NNTCACACAGGAAACCTCTAGAATG }\end{array}$ & BioBrick & $\begin{array}{l}\text { mTagBFP } \\
\text { EYFP }\end{array}$ & $\begin{array}{l}{[6]} \\
{[6]}\end{array}$ \\
\hline $\begin{array}{l}\text { BBa_B0032 } \\
(\mathrm{N})_{n} \bar{T} C A C A C A G G A A A G(N)_{n} \text { ATG } \\
\text { AGATCACACAGGAAAGTACTAGATG } \\
\text { NNNTCACACAGGAAAGTCTAGAATG }\end{array}$ & BioBrick & $\begin{array}{l}\text { GFPmut3b } \\
\text { mTagBFP } \\
\text { EYFP }\end{array}$ & $\begin{array}{l}{[4]} \\
{[6]} \\
{[6]}\end{array}$ \\
\hline $\begin{array}{l}\text { BBa_B0033 } \\
\text { CTAGATCACACAGGACTACTAGATG } \\
\text { NNNNNTCACACAGGACTCTAGAATG }\end{array}$ & BioBrick & $\begin{array}{l}\text { mTagBFP } \\
\text { EYFP }\end{array}$ & $\begin{array}{l}{[6]} \\
{[6]}\end{array}$ \\
\hline $\begin{array}{l}\text { BBa_B0034 } \\
\left.(\mathrm{N})_{n} \text { AAAGAGGAGAAA(N)}\right)_{n} \text { ATG } \\
\text { TAGAAAAGAGGAGAAATACTAGATG } \\
\text { NNNNAAAGAGGAGAAATCTAGAATG }\end{array}$ & BioBrick & $\begin{array}{l}\text { GFPmut3b } \\
\text { mTagBFP } \\
\text { EYFP }\end{array}$ & $\begin{array}{l}{[4]} \\
{[6]} \\
{[6]}\end{array}$ \\
\hline $\begin{array}{l}\text { BBa_B0035 } \\
\text { GAATTAAAGAGGAGAATACTAGATG } \\
\text { NNATTAAAGAGGAGAATCTAGAATG }\end{array}$ & BioBrick & $\begin{array}{l}\text { mTagBFP } \\
\text { EYFP }\end{array}$ & $\begin{array}{l}{[6]} \\
{[6]}\end{array}$ \\
\hline $\begin{array}{l}\text { BBa_B0064 } \\
\text { TAGAAAAGAGGGGAAATACTAGATG } \\
\text { NNNNAAAGAGGGGAAATCTAGAATG }\end{array}$ & BioBrick & $\begin{array}{l}\text { mTagBFP } \\
\text { EYFP }\end{array}$ & $\begin{array}{l}{[6]} \\
{[6]}\end{array}$ \\
\hline $\begin{array}{l}\text { psbA2 RBS } \\
\text { AGACATAAGGAATTATTACTAGATG } \\
\text { NNNCATAAGGAATTATTCTAGAATG }\end{array}$ & $p s b A 2^{a}$ & $\begin{array}{l}\text { mTagBFP } \\
\text { EYFP }\end{array}$ & $\begin{array}{l}{[6]} \\
{[6]}\end{array}$ \\
\hline $\begin{array}{l}r b c L \text { RBS } \\
\text { AGATTATGGAGGACTGTACTAGATG } \\
\text { NNNTTATGGGGACTGTCTAGAATG }\end{array}$ & $r b c L^{\mathrm{a}}$ & $\begin{array}{l}\text { mTagBFP } \\
\text { EYFP }\end{array}$ & $\begin{array}{l}{[6]} \\
{[6]}\end{array}$ \\
\hline $\begin{array}{l}\text { RBS* } \\
\text { NNNNNNTAGTGGAGGTNNNNNNATG } \\
\text { TCTAGATAGTGGAGGTTACTAGATG } \\
\text { NNNNNNTAGTGGAGGTTCTAGAATG }\end{array}$ & A synthetic RBS ${ }^{a}$ & $\begin{array}{l}\text { GFPmut3b } \\
\text { mTagBFP } \\
\text { EYFP }\end{array}$ & $\begin{array}{l}{[4]} \\
{[6]} \\
{[6]}\end{array}$ \\
\hline $\begin{array}{l}\text { Unnamed } \\
\text { NAVGAAGGGGTNNNNNNNNNNNNATG }\end{array}$ & A synthetic RBS & eCFP and YFP & {$[5]$} \\
\hline $\begin{array}{l}\text { RBSV4 } \\
\text { AAAATAAAGAAGGAGGAACAGCATG }\end{array}$ & A synthetic RBS & Synthetic efe & [7] \\
\hline
\end{tabular}

The sequences (22 bp upstream from the initiation codon) are presented in $5^{\prime}-3^{\prime}$ direction, with the core SD consensus sequence GGAGG underlined and ATG shown in bold. The nucleotides that have not been specified in the corresponding publications are denoted as N. Comparative studies in which the RBS is not clearly distinguished from the context of the promoter have not been included

a Indicates that the corresponding gene has been used also as the source of RBS sequence in this study

The objective of this study was to expand the current knowledge on the factors influencing translational efficiency in the cyanobacterium Synechocystis. This was to be accomplished by systematic quantitative comparison of a set of selected RBS sequences in vivo in order to (i) find specific sequences allowing the highest translational efficiencies, (ii) define a series of RBSs covering a dynamic range of different expression levels, and (iii) evaluate the extent to which the nucleotide sequence of the target gene affects the outcome. The aim was to compile a library of alternative expression constructs harboring an array of RBSs fused with fluorescent reporter genes sYFP2 and GFPmut3b (YFP and GFP from here on, respectively) as quantitative indicators of translation efficiency, followed by generation and fluorometric characterization of the corresponding Synechocystis strains. The experimental study was to be complemented by comparative bioinformatic analysis to correlate between the RBS sequences and obtained expression levels, and to predict possible secondary structures between the coding 
sequence and the upstream region to explain observed differences.

\section{Results}

\section{Selecting RBSs for in vivo comparison in Synechocystis}

Thirteen different RBS sequences were selected for the comparative study to determine quantitative differences in translation efficiencies in Synechocystis (Table 2). Six of the sequences form a degenerative series that has previously been evaluated in E. coli, where they span an expression level range of several magnitudes $(\mathrm{A}-\mathrm{E}, \mathrm{Z}$; in decreasing order of efficiency) $[3,12]$. The remaining seven RBS sequences derived either directly from native highly expressed cyanobacterial genes (Table 2; S2-S5), or from expression constructs designed for Synechocystis (Table 2; S1, S6, S7), and had not previously been systematically compared for efficiency. The RBS sequences were composed of 12-22 bp variable region immediately upstream from the start codon ATG (Table 2), preceded by an invariant $5^{\prime}$ upstream spacer-insulator region [3]. The sequences were ordered as synthetic DNA fragments, flanked by specific restriction sites, which could be directly used for the generation of the expression construct library.

\section{Generation of the expression strains for evaluating the RBS efficiencies}

In order to evaluate the efficiency of the designed RBSs in Synechocystis in vivo, each of the sequences was first fused with a gene coding for the fluorescent reporter protein YFP [18] and a codon-optimized counterpart GFP (Additional file 1) [19]. These two reporters were specifically designed to have low nucleotide sequence similarity between one another to study the influence of the genetic context (i.e. the combined effect of the RBSs with the coding sequence) on the outcome. To enable expression in Synechocystis, the fragments were transferred into a pDF-lac [20]-derived expression plasmid pDF-lac2, under the control of the IPTG inducible Lac promoter variant $\mathrm{P}_{\mathrm{AllacO}-1}$. The generated 26 plasmid constructs (Additional file 2) were then transformed into Synechocystis, and the resulting antibiotic resistant strains were verified by colony PCR (Additional file 3: A, B).

Table 2 The RBS sequences compared in this study

\begin{tabular}{|c|c|c|}
\hline Name and sequence & Gene/source & Genebank ID/references \\
\hline $\begin{array}{l}\text { S1 } \\
\text { CTAGAGTAGTGGAGGTTACTAGATG }\end{array}$ & Synthetic RBS based on Synechocystis anti-SD & {$[4,25]$} \\
\hline $\begin{array}{l}\text { S2 } \\
\text { AATATAAGTAGGAGATAAAAACATG }\end{array}$ & cpcB from Synechococcus sp. PCC 7002 & СР000951.1 \\
\hline $\begin{array}{l}\text { S3 } \\
\text { AGTCAAGTAGGAGATTAATTCAATG }\end{array}$ & s/11577, cpcB from Synechocystis & U34930.1 \\
\hline $\begin{array}{l}\text { S4 } \\
\text { ATACATAAGGAATTATAACCAAATG }\end{array}$ & slr1131, psbA2 from Synechocystis & X13547.1 \\
\hline $\begin{array}{l}\text { S5 } \\
\text { TGTTTATGGAGGACTGACCTAGATG }\end{array}$ & slr0090, rbcL from Synechocystis & X65960.1 \\
\hline $\begin{array}{l}\text { S6 } \\
\text { TAGCCTAGGAGGAGGAAAAATCATG }\end{array}$ & Synthetic RBS & Unpublished construct \\
\hline $\begin{array}{l}\text { S7 } \\
\text { CATTAAAGAGGAGAAAGGTACCATG }\end{array}$ & pDF-lac & {$[20]$} \\
\hline $\begin{array}{l}\text { A } \\
\text { AACAAAATGAGGAGGTACTGAGATG }\end{array}$ & Adapted from Salis et al. [12] & [3] \\
\hline $\begin{array}{l}\text { B } \\
\text { TTTAACTTTAAGGAGGTTTGGAATG }\end{array}$ & Adapted from Salis et al. [12] & [3] \\
\hline $\begin{array}{l}\text { C } \\
\text { ACTTTAAAGTTAAGAGGCAAGAATG }\end{array}$ & Adapted from Salis et al. [12] & [3] \\
\hline $\begin{array}{l}\text { D } \\
\text { TTTATAAGCAGGACCGGCGGCGATG }\end{array}$ & Adapted from Salis et al. [12] & {$[3]$} \\
\hline $\begin{array}{l}\text { E } \\
\text { TAACTTTATTCGCAGGGGGAAGATG }\end{array}$ & Adapted from Salis et al. [12] & [3] \\
\hline $\begin{array}{l}\text { Z } \\
\text { TTTAACTTTACACCATACACTGATG }\end{array}$ & Adapted from Salis et al. [12] & [3] \\
\hline
\end{tabular}

S2-S5 originate directly from the native cyanobacterial genes and correspond to the NCBI Genbank sequences (22 bp preceding the start codon), while S1 and S6-S7 derive from expression constructs designed earlier for Synechocystis sp. PCC 6803. RBS A-E and Z represent sequences which have earlier been characterized on E. coli. The core SD consensus sequence GGAGG has been underlined, start codon ATG shown in bold, and the upstream insulator sequence identical in all constructs shown in italics 


\section{Analytical set-up for the comparative analysis of RBSs}

To confirm the function of the expression systems and to optimize the analytical set-up, the 26 generated Synechocystis strains were subjected to a series of fluorescence spectroscopy measurements. Time course analysis between 0 and $6 \mathrm{~h}$ after induction (Additional file 4) showed a clear fluorescence response for majority of the strains in respect to YFP and GFP measured at $495 \mathrm{~nm}(\mathrm{ex}.) / 535 \mathrm{~nm}(\mathrm{em}$.$) and 485 \mathrm{~nm}(\mathrm{ex}.) / 525 \mathrm{~nm}$ (em.), respectively. Based on the response profiles, the 6-h mark was selected as the default time-point for the quantitative comparison of the strains in successive experiments, due to sufficiently broad intensity range and signal levels which remained clearly below the saturation limit. Comparison of the induced and uninduced fluorescent profiles showed that the promoter $\mathrm{P}_{\mathrm{A} 1 \mathrm{lacO}-1}$ was adequately repressed under the experimental conditions (Additional file 4; see dotted lines), confirming that unspecific background fluorescence did not have any apparent effect on the data interpretation. In addition, the differences in growth of the parallel strains appeared to be insignificant and without direct correlation with the observed expression levels (Additional file 5), yet to ensure the validity of the comparison, the fluorescence signals were in each case normalized to $\mathrm{OD}_{750 \mathrm{~nm}}$.

\section{System validation by two parallel analytical approaches}

The 26 generated Synechocystis strains with 13 distinct RBS sequences regulating the translation of YFP and GFP were subjected to two independent rounds of quantitative analysis in order to confirm the validity of the expression-level comparison. In the first approach (i.e. full dataset), each of the 13 strains was characterized individually on separate days, using six biological replicates with three technical replicates $(n=18)$, for both YFP (Fig. 1a) and GFP (Fig. 1b). In the second approach (i.e. one-day dataset) all the 13 strains were analyzed in parallel on the same day but with only three technical replicates $(n=3)$ for YFP (Fig. 2a) and GFP (Fig. 2b). The primary observation was that, besides the broad range of expression levels, the variation between the biological replicates within each full dataset was negligible (Fig. 1 and Additional file 4). This indicated low clonal variation and high reproducibility, which were essential for meaningful statistical evaluation of the RBSs. Comparison of each full dataset (Fig. 1) with the corresponding one-day dataset (Fig. 2), on the other hand, showed that the RBS-specific expression profiles were highly similar between the two approaches even by mere visual evaluation. Statistical analysis conducted by two independent methods further verified high correlation between the full dataset and the one-day dataset with high confidence ( $\mathrm{p}$ values under 0.001) (Table 3). This demonstrated that the two alternative experimental approaches resulted in a very similar outcome, and individually represented the relative RBS-specific expression trends to a reliable degree. For maximal statistical significance, the full datasets with six biological replicates (Fig. 1) were used for the subsequent rounds of analysis.
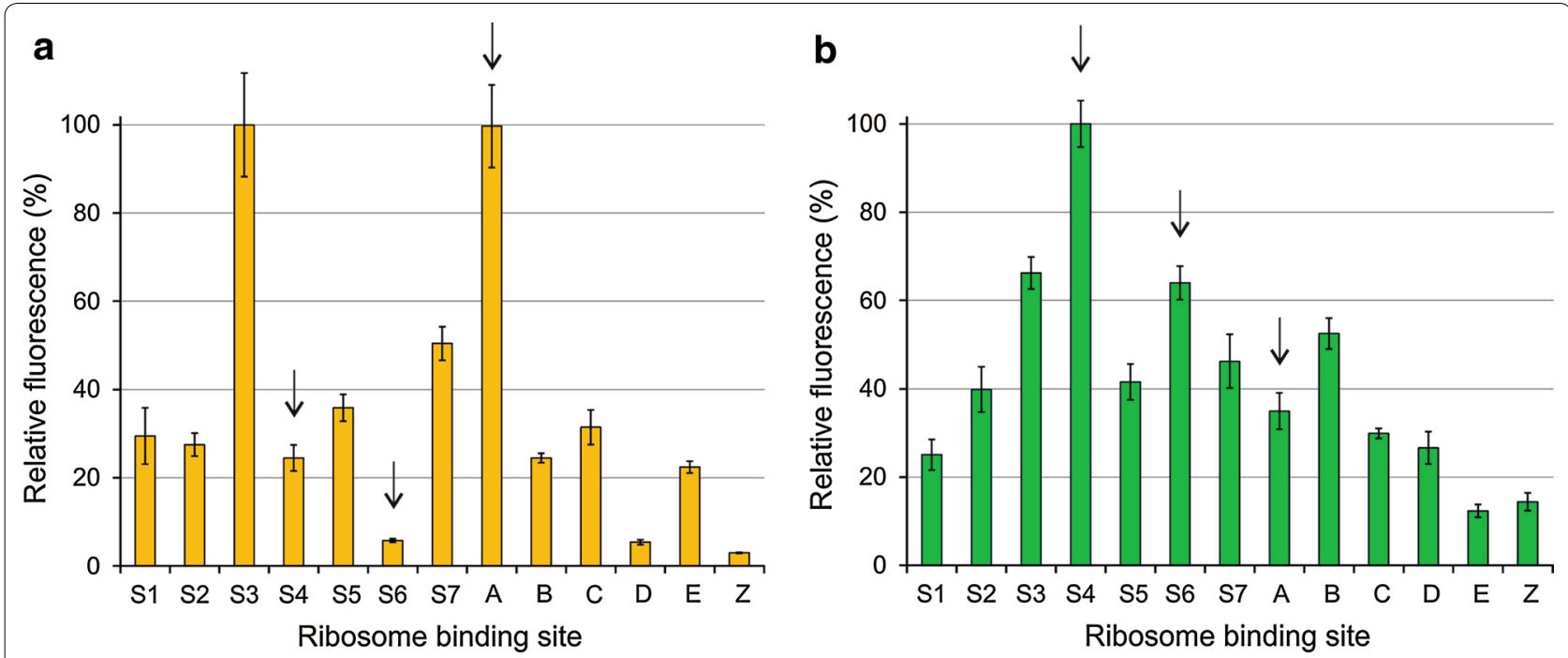

Fig. 1 Relative fluorescence (full dataset) representing the translational efficiencies of the 13 RBSs in engineered Synechocystis sp. PCC 6803 strains. Two alternative fluorescence reporters used in the study were $\mathbf{a}$ sYFP2 and $\mathbf{b}$ GFPmut3b, measured from intact cells at $6 \mathrm{~h}$ after induction. The bars showing the average relative fluorescence values (\%) and standard deviations were calculated from six parallel biological replicates and three technical replicates $(n=18)$ normalized to $\mathrm{OD}_{750 \mathrm{~nm}}$. The arrows indicate the RBSs which differ the most between the two datasets 

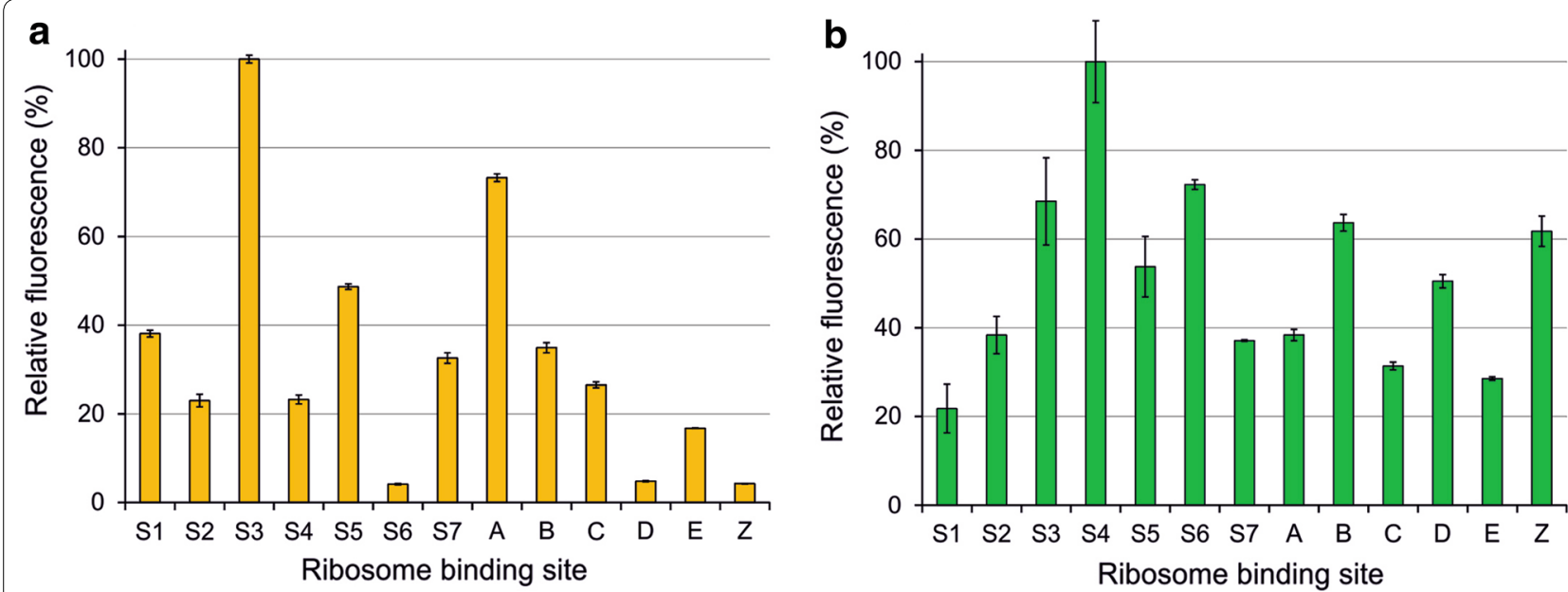

Fig. 2 Relative fluorescence (one-day dataset) representing translational efficiencies of the 13 RBSs in engineered Synechocystis sp. PCC 6803 strains. Two alternative fluorescence reporters used in the study were $\mathbf{a}$ sYFP2 and $\mathbf{b}$ GFPmut $3 \mathrm{~b}$, measured from intact cells at $6 \mathrm{~h}$ after induction. The bars showing the average relative fluorescence values (\%) and standard deviations were calculated from one biological replicate and three technical replicates $(n=3)$ and normalized to $\mathrm{OD}_{750 \mathrm{~nm}}$

Table 3 Statistical comparison between the full dataset and the one-day dataset of SYFP2 and GFPmut3b fluorescence profiles using Pearson correlation coefficient and Spearman's rank correlation coefficient

\begin{tabular}{|c|c|c|c|c|}
\hline \multirow[b]{2}{*}{ Method } & \multicolumn{2}{|c|}{$\begin{array}{l}\text { YFP: full dataset vs 1-day } \\
\text { dataset }\end{array}$} & \multicolumn{2}{|c|}{$\begin{array}{l}\text { GFP: full dataset vs } \\
\text { 1-day dataset }\end{array}$} \\
\hline & correlation & $p$-values & correlation & $\mathrm{p}$-values \\
\hline Pearson & 0.942 & $<0.0001$ & 0.801 & 0.001 \\
\hline Spearman & 0.915 & 0.0002 & 0.918 & $<0.0001$ \\
\hline
\end{tabular}

The YFP and GFP expression profiles are clearly distinct

The YFP and GFP genes used in this study did not share any significant nucleotide sequence similarity due to GFP codon optimization, and consequently, could be used as representatives of unrelated target genes to study the effect of the downstream coding region on the RBS-specific expression. Comparison between the YFP (Fig. 1a) and GFP fluorescence datasets (Fig. 1b) revealed distinct divergence in the overall shapes of the profiles, reflecting pronounced gene-specific differences in the expression of different RBS strains. As further verified by the low statistical correlation between the YFP and GFP datasets (Table 4), the expression levels were clearly not determined by the specific RBS sequences alone, but also significantly affected by the downstream gene sequence. At the level of individual strains, while the relative performance of many of the 13 RBSs appeared to be similar with YFP and GFP (Fig. 1), the translational efficiency recorded for several specific constructs was dramatically affected by the downstream region. The most distinct differences were observed for RBSs S4, S6 and A (Fig. 1; see
Table 4 Statistical comparison between the sYFP2 and GFPmut3b fluorescence profiles (full dataset) using Pearson correlation coefficient and Spearman's rank correlation coefficient

\begin{tabular}{llllll}
\hline Method & Full dataset & & & \multicolumn{2}{l}{$\begin{array}{l}\text { Full dataset excluding } \\
\text { S4 }+\mathbf{S 6}+\mathbf{A}\end{array}$} \\
\cline { 2 - 3 } \cline { 6 - 7 } & YFP vs GFP & & & YFP vs GFP & \\
\cline { 2 - 3 } \cline { 5 - 6 } & Correlation & p value & & Correlation & p value \\
\hline Pearson & 0.186 & 0.346 & & 0.781 & 0.008 \\
Spearman & 0.543 & 0.243 & & 0.697 & 0.029 \\
\hline
\end{tabular}

The table shows the values calculated based on the entire dataset, and after the exclusion of the most divergent RBSs (S4, S6 and A)

arrows) which performed almost in an opposite manner in respect to the expression of YFP and GFP. Notably, exclusion of these RBSs from the comparison (Table 4) markedly improved the statistical correlation and p-values, reflecting the degree of underlying similarity between the YFP and GFP profiles, and ultimately in the efficiency of individual RBSs which may be easily masked by context-dependent effects.

\section{Confirmation of the functional context dependence}

To further assess the connection between gene sequence and translation efficiency, six RBSs (S1, S3, S5, S7, E, Z) with relatively uniform expression profiles between YFP (Fig. 1a) and GFP (Fig. 1b) were selected for an independent analysis using ethylene-forming enzyme (E.C. 1.14.11.34) as an alternative quantitative reporter. This enzyme (encoded by efe from Pseudomonas syringae) enables the conversion of intracellular 2-oxoglutarate 
into ethylene [21], which can be quantitatively measured from the headspace of sealed culture vials by gas chromatography $(\mathrm{GC})$. The expression plasmids carrying the efe gene under the control of the six alternative RBSs were assembled the same way as the fluorescent reporter constructs, transformed into Synechocystis, and verified by colony PCR (Additional file 3: C). The analysis revealed that ethylene production between parallel biological replicates was highly reproducible, and enabled RBS-specific quantitative evaluation between the strains in analogy to the use of the fluorescent reporters (Fig. 3a). While the relative signal intensities measured for most RBSs followed the trends observed for YFP and GFP (Fig. 1), the performance of S3 was especially poor with efe. In parallel, the relative activity of S5 appeared higher than expected based on YFP and GFP profiles used for the comparison.

\section{Sequence analysis of the RBSs fails to predict performance in Synechocystis}

Based on the obtained activity data, the recorded efficiencies of the RBSs were complemented by in silico analysis to pinpoint potential nucleotide sequence-level determinants which would explain the most significant RBSspecific variation between the datasets. Specifically, the objective was to find correlation between putative mRNA secondary structure formation and reduced expression levels between the YFP and GFP systems for the RBSs S4, S6 and A (representing divergence) and the remaining RBSs (representing similarity). The comparison carried out with two alternative on-line prediction tools clearly revealed that the minimum free energies (MFE) calculated for the regions around the start codon $(-25$ to +35 ) (Additional file 6: A, B) or the entire sequences (Additional file 6: C, D) do not provide any clear indication of unfavorable sequence-level interactions which would explain the functional divergence. In addition, estimation of the translation efficiencies based on the 13 RBS sequences using the Salis calculator (Fig. 3b) and UTR Designer (Additional file 7) resulted in similar outputs that differed significantly from the measured expression profiles, supporting the view that that the reverse engineering approach does not allow relevant predictions which would correlate with the experimental findings in Synechocystis.

\section{Discussion}

There is a profound need to establish new industrial solutions for sustainable large-scale production of different carbon-based chemicals to reduce our current dependency on petroleum-derived products. In this context, photosynthetic microorganisms have already been recognized at EU-level as part of emerging future technologies [22], as potential biotechnological hosts for generating desired target chemicals directly from atmospheric $\mathrm{CO}_{2}$. This study addressed the apparent need for more robust molecular biology tools and identification of the associated bottlenecks in cyanobacterial engineering, which have been recognized as major limitations in the development of commercially viable systems [22]. Specifically,
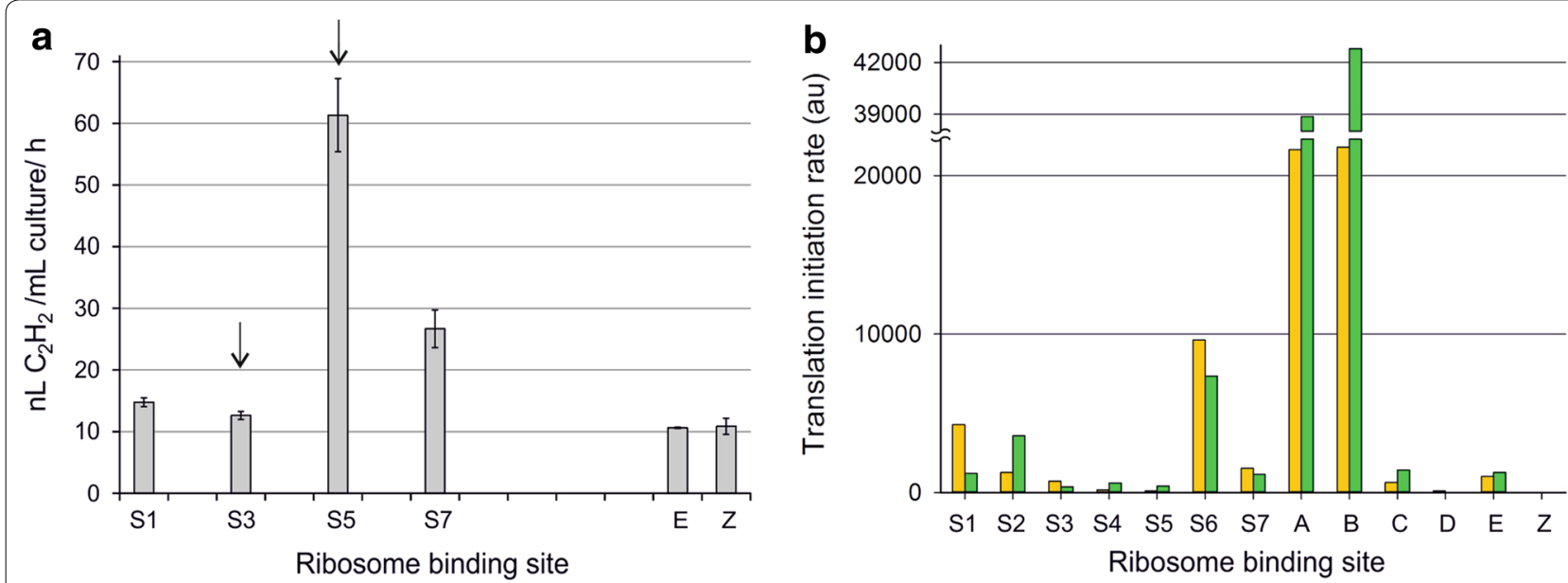

Fig. 3 Evaluation of the translational efficiencies of selected RBS sequences based on a ethylene productivity of six designed strains expressing ethylene-forming enzyme measured from the headspace at $4 \mathrm{~h}$ after induction. The bars showing the average ethylene productivity $\left(n L \mathrm{C}_{2} \mathrm{H}_{2} / \mathrm{ml}\right.$ culture/h) and standard deviations were calculated from three biological replicates $(n=3)$ and normalized to $\mathrm{OD}_{750 \mathrm{~nm}}$. The arrows indicate the RBSs which differ the most in comparison to the fluorescent datasets in Fig. 1. b Salis calculator prediction for the translation initiation rate using the nucleotide sequence around the start codon $(-25$ to +35$)$ as input, displaying predicted translation initiation rates for all the RBSs in combination with the two fluorescent reporters sYFP2 (yellow) and GFPmut3b (green) 
the work focused on the systematic functional comparison of ribosome binding sites in the cyanobacterial host Synechocystis sp. PCC 6803, with the objective to expand the prospects for targeted use of RBSs as translational regulatory elements in sophisticated rational pathway design. From engineering viewpoint, the information provided in this study sets a basis for regulating the relative expression levels of individual genes in polycistronic operons in Synechocystis, which is essential for the functional optimization of heterologous pathways in future applications, as simple expression level maximization does not necessarily ensure the most efficient flux through the subsequent enzyme-catalyzed steps [3]. In addition to providing dynamic data on the function of 13 selected RBSs in vivo, the work underlines the severe constraints related to gene-specific interactions, and the importance of finding efficient strategies for expression construct assembly to meet the demands for increased complexity, predictability and preparative throughput.

Cyanobacterial engineering has traditionally relied on conventional cloning strategies which are relatively rigid for generating multiple parallel variations of constructs, or multi-gene operons which require the assembly of many different components in alternate arrangements. In addition, due to the lack of congruence between the approaches and a limited palette of validated speciesspecific genetic elements, finding reliable comparable information on the most the optimal components may be challenging. To overcome the preparative constraints and facilitate the design phase, we adapted a modular cloning strategy, in which compatible genetic elements can be fused together in any order via iterative subcloning steps based on restriction site recycling [3]. Besides enabling the assembly of 32 alternative Synechocystis-compatible reporter constructs with relative ease, the system can now be used for fusing the evaluated RBS sequences in the library with any target gene of choice, and further, allows the individual RBS-gene fragments to be joined into polycistronic operons. Importantly, the generated library together with the construction platform provides the means for pathway optimization in Synechocystis, as the expression level of individual proteins can now be modulated at translational level by selecting appropriate combinations of RBSs in front of the genes. Besides the rational approach, the system also is suitable for randomized RBS selection [3], which may be used for optimizing multi-gene operons even if the relative efficiency of the RBSs isn't precisely known, yet requires access to a high-throughput screening system to identify the bestperforming clones. For the future development in the field, it is also important not to be confined to a single cyanobacterial strain, and to be able to apply the developed synthetic biology tools for alternate hosts such as
Synechococcus elongatus PCC 7942 or Synechococcus sp. PCC 7002 which may be better suited for a specific purpose. As demonstrated in the current work, the assembly system can be adapted for any prokaryotic expression system, simply by using a suitable host-specific vector at the last subcloning step.

Based on the acquired data from multiple parallel replicates and independently produced datasets, the results were highly reproducible, and allowed direct quantitative comparison of the translation efficiencies of the different RBS sequences. As the primary observation, the 13 characterized RBSs produced a relatively broad range of expression levels for both fluorescent reporters (Fig. 1), thus allowing access to dynamic translation-level regulation in engineered systems in Synechocystis. As a downside from engineering viewpoint, however, the nucleotide sequence of the target gene has potentially a profound effect on the expression level, as seen in the low correlation between the signal profiles produced by YFP and the codon-optimized GFP (Table 4). The observation is not unexpected as such, and the phenomenon has been encountered earlier in cyanobacteria, but up until now the extent to which this may interfere with the function of engineered cyanobacterial expression systems has remained elusive. Generally, the effect is caused by mRNA secondary structure formation between a specific RBS and the coding region sequence, which may affect the ribosome recognition or efficient initiation complex formation. Two of the RBSs which functioned in the most unpredictable manner in the test set-up (Fig. 1; see arrows) are S4 from Synechocystis psbA2 gene encoding the photosystem II reaction center protein D1, which has been used in many engineering applications, and A which has been reported to give the highest translation levels in E. coli $[3,12]$. While the results show that S4 and A would not have been optimal choices for maximizing the expression of the YFP and GFP, respectively, it is recognized that the interactions are in each case determined by the associated combined effects with the following gene sequence and not the specific RBS sequences per se. This was also corroborated in the independent analytical trial where the RBS S3 from $c p c B$ encoding the phycocyanin subunit $\beta$-one of the most efficient RBSs in Synechocystis [23] used in a number of expression systems-functioned very poorly in context with an alternate heterologous reporter gene efe (Fig. 3a). The complication is further emphasized by the inability of currently available in silico prediction tools to approximate the relative RBS efficiencies for Synechocystis (Fig. 3b and Additional file 7) or to identify potentially interfering mRNA secondary structures in specified RBSs which perform in a context-dependent manner in the set-up (Additional file 6). This discrepancy is expected 
to result from, at least in part, a longer optimal distance between the SD core sequence and the start codon, as well as the organism-specific culture conditions such as growth temperature, between Synechocystis and E. coli for which the system has been optimized for [24]. However, a significant increase was observed in the correlation between the recorded YFP and GFP full fluorescence datasets in response to the exclusion of the most inconsistent RBSs from the comparison (Table 4), which indicates that (i) the expression profiles are not random but (ii) clearly follow an underlying pattern which may be masked by gene-specific interactions. This suggests that the obtained datasets can be used to a certain extent to predict the relative expression efficiencies (i.e. evaluating the potential), with the caution that the true performance of a specific RBS with a given gene would also require experimental verification. Although exact numerical comparison may be meaningless due to the observed context-dependence, the RBSs can be at least roughly divided into groups based on apparent high, moderate and low activity, which may serve as an initial guideline for selecting appropriate control elements for engineering work.

As seen from the combined YFP and GFP data, the RBSs S3, S4 and A (Fig. 1 and Table 2) appear to have potential as prominent high-activity elements useful for maximizing translational efficiency in Synechocystis. This is not unexpected since $\mathrm{S} 3(c p c B)$ and $\mathrm{S} 4$ ( $p s b A 2)$ derive from genes which are exceptionally highly expressed in the native context, while A performs at the most optimal efficiency in heterologous systems in E. coli. The remaining cyanobacterial RBSs (or RBSs used in cyanobacteria), S1, S2, S5 and S7 (Fig. 1 and Table 2), displayed activities between 25 and $50 \%$ of the maximum capacity in the experimental set-up. These efficiencies appear relatively moderate, considering that $\mathrm{S} 1$ represents a sequence specifically optimized for the host [4, 25], S2 originates from the homologous $c p c B$ gene in Synechococcus sp. PCC 7002, and S5 is from $r b c L$ encoding for the large subunit of Rubisco in Synechocystis, but again, may suffer from the context-dependent effects. RBS S7 from the expression plasmid pDF-lac [20], which has been applied in various studies in Synechocystis with generally high expression profiles, performed at about half of the maximum activity for all the three reporter systems. The RBSs A-Z spanned the entire activity range and roughly followed the order as reported for $E$. coli, although genespecific deviation could be observed (Fig. 1). In order to employ the available potential of using the RBSs for reliable translational tuning in Synechocystis, it is clear that that new approaches are needed to decouple UTR region and coding sequence interactions. One possible strategy would be to design additional downstream insulator sequences to minimize potential interfering secondary structure formation between the RBS and the immediate downstream region [26] to reduce the observed genedependent effects in cyanobacterial expression systems.

\section{Conclusions}

A set of 13 different RBS sequences characterized in this work can be used for the regulation of the protein expression efficiency at a wide dynamic range in the cyanobacterium Synechocystis sp. PCC 6803. However, the absolute activity of a specific RBS is difficult to determine due to sequence-specific effects caused by the downstream target gene, which may result in significantly decreased translation efficiencies. Despite this context dependence, there appears to be a level of similarity between the underlying expression profiles, which can be used as an indicator of the potential performance of the alternative RBSs in engineered systems. Due to the limited availability of associated functional information in cyanobacteria, reliable prediction of potential interactions that may reduce translation efficiency is difficult, and calls for new design strategies to minimize unwanted interactions between the RBS and the downstream target gene.

\section{Methods}

\section{Enzymes and reagents}

The restriction enzymes, T4 DNA ligase and DNA polymerase used in this work were purchased from New England BioLabs (USA) or from ThermoFischer-Scientific (USA). Commercial Qiagen (Germany) kits were used for plasmid isolation (QIAprep Spin miniprep kit) and gel extraction (QIAquick, gel extraction kit). Oligonucleotides were ordered from Eurofins MWG Operon (Germany), and larger gene fragments from GenScript (USA). All other chemicals were purchased from Sigma-Aldrich (USA), unless mentioned otherwise.

\section{Bacterial strains and standard growth conditions}

Escherichia coli strain DH5 $\alpha$ was used for plasmid propagation and selection in the preparative molecular biology steps. The cells were grown in Luria-Bertani (LB) medium at $37{ }^{\circ} \mathrm{C}$ in a shaker at $150-200 \mathrm{rpm}$ or on the solid LB plates containing 1.5\% (w/v) agar. When necessary, LB medium was supplemented with appropriate antibiotics at concentrations $50 \mu \mathrm{g} / \mathrm{ml}$ spectinomycin $(\mathrm{Sp})$ and $34 \mu \mathrm{g} / \mathrm{ml}$ chloramphenicol $(\mathrm{Cm})$.

A glucose tolerant substrain of Synechocystis sp. PCC 6803 [27] obtained originally from Professor Aaron Kaplan (Hebrew University of Jerusalem, IL) was used for all the cyanobacterial experiments. The cells were grown in $25-50 \mathrm{ml}$ Erlenmeyer flasks in liquid BG-11 medium buffered with $20 \mathrm{mM}$ TES-KOH (pH 8.0) [28] 
with supplemented $25 \mu \mathrm{g} / \mathrm{ml} \mathrm{Sp}$ and $10 \mu \mathrm{g} / \mathrm{ml} \mathrm{Cm}$ to maintain transformant selection pressure throughout all cultivations. The cultures were incubated at $30{ }^{\circ} \mathrm{C}$ in $\sim 120 \mathrm{rpm}$ orbital shaking under continuous light of 20-50 $\mu$ mol photons $\mathrm{m}^{-2} \mathrm{~s}^{-1}$ under $1 \% \mathrm{CO}_{2}$ atmosphere (MLR-351 growth chamber Sanyo, Japan) or under ambient $\mathrm{CO}_{2}$ (Algaetron 230 growth chamber, Photon Systems Instruments, CZ). Solid plate cultivations were conducted on BG-11 plates containing additional 1.5\% $(\mathrm{w} / \mathrm{v})$ Bactoagar (Difco, USA) and 0.3\% (w/v) sodium thiosulfate under corresponding conditions (MLR-351, Sanyo).

\section{Generating the RBS library}

Six of the RBS fragments used in the study (A-E, Z) (Table 2) were provided by Professor Ron Milo as corresponding pNiv constructs [3]. The remaining RBS fragments were designed to carry a 22 bp variable region immediately upstream the start codon, corresponding to the exact native target gene sequences acquired from NCBI GenBank (S2-S5) or existing expression construct sequences (S1, S6-S7) (Table 2), and preceded by a 27 bp invariable upstream insulator sequence common to all the constructs (5'-TAATAGAAATAATTTTGTTTA ACTTTA- $3^{\prime}$ ). These fragments were ordered as synthesized complementary single-stranded oligonucleotides (MWG, Germany), mixed in pairs, and subcloned into pNiv (SpeI-NsiI). The resulting library was verified by sequencing using the primer $5^{\prime}$-CTTCCTGTTAGTTAG TTACTTAAGCTCGG - $3^{\prime}$.

\section{Generation of the reporter genes}

The three reporter genes, sYFP2 [18], GFPmut3b [19] and efe [21] used in the study were ordered as synthetic fragments (GenScipt). The genes for sYFP2 (GenBank ID DQ092361.1) and efe (GenBank ID AF101058.1) were based on the original NCBI nucleotide sequences, while GFPmut3b (GenBank ID AAB51348.1) was codon-optimized for Synechosystis (Additional file 1). The fragments were designed as chloramphenicol resistance cassette $\left(\mathrm{Cm}^{\mathrm{R}}\right)$ fusions [3] flanked by restriction sites NsiI and XhoI, and avoiding the restriction sites EcoRI, SpeI, NheI, and SalI within the coding regions to ensure compatibility with the assembly system [3].

\section{Assembly of the RBS-reporter constructs}

The construct assembly system adapted for fusing the RBS sequences with the reporter genes was based on a modular cloning strategy described by Zelcbuch et al. [3]. The genes (synthesized as $\mathrm{Cm}^{\mathrm{R}}$-fusions for selection) were first subcloned directly downstream of each of the 13 RBS sequences in the pNiv carrier plasmids (NsiIXhoI), followed by the transfer of the combined fragments into an expression plasmid pDF-lac2 (SpeI-SalI) specifically designed for the purpose. All the plasmids generated in this study are listed in Additional file 2.

\section{Assembly of a compatible expression plasmid pDF-lac2}

The expression plasmid used for characterizing the RBSs in Synechocystis was generated from the shuttle vector pDF-lac [20] by replacing the SpeI-SalI region of the plasmid spanning the promoter region with a compatible synthetic NheI-SalI fragment (see sequence below; restriction sites NheI, SpeI and SalI underlined, respectively). This modification placed the SpeI site in between the promoter and the existing SalI site, thus allowing direct transfer of fragments from the pNiv assembly plasmid (SpeI-SalI) under the control of the promoter $\mathrm{P}_{\mathrm{AllacO}-1}$.

5'-GCTAGCGAGCGGATACATATTTGAATGTAT TTAGAAAAATAAACAAATAGGGGTTCCGCGCAC ATTTCCCCGAAAAGTGCCACCTGACGTCTAAGA AACCATTAT TATCATGACATTAACCTATAAAAA TAGGCGTATCACGAGGCCCTTTCGTCTTCACCT CGAGAAATTTATCAAAAAGAGTGTTGACTTGTG AGCGGATAACAATGATACTTAGATTCAATTGTG AGCGGATAACAATTTCACACAGAATTCATTAAAG

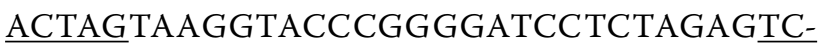
GAC-3'.

\section{Generation of the Synechocystis strains for RBS comparison} The generated pDF-lac2-based expression constructs (Additional file 2) were transformed into the WT Synechocystis [29] and plated on BG11 plates supplemented with increasing amounts of $\mathrm{Sp}$ and $\mathrm{Cm}$. Antibiotic resistant clones were transferred onto secondary plates, confirmed by colony PCR, and stored at $-80{ }^{\circ} \mathrm{C}$ with $7.5 \%$ DMSO until characterization.

\section{Quantitative fluorescence analysis of the Synechocystis strains expressing YFP and GFP}

Quantitative fluorescent analysis of the Synechocystis strains expressing sYFP2 and GFPmut3b under the control of the alternative RBSs was carried out using a Tecan microplate reader (Tecan infinite 200 PRO) with $495 \mathrm{~nm}$ (ex)/535 nm (em) and $485 \mathrm{~nm}(\mathrm{ex}) / 525 \mathrm{~nm}(\mathrm{em})$, respectively. The analysis was conducted on intact cells (culture 
volume $150 \mu \mathrm{l}$ ) on 96 -well black clear bottom polystyrene plates (Costar Corning, USA) for six biological replicates (full dataset) or one representative clone (1-day dataset) with three technical replicates in each case. To allow reproducible comparison, the pre-cultures inoculated from freshly prepared plates were first diluted to $\mathrm{OD}_{750}$ 0.28 (Thermo Scientific GENESYS 10S UV-Vis spectrophotometer), and grown under ambient $\mathrm{CO}_{2}$ for $\sim 18 \mathrm{~h}$. At $\mathrm{OD}_{750} \sim 0.5$ the cultures were induced by the addition of $1 \mathrm{mM}$ of isopropyl- $\beta$-D-thiogalactopyranoside (IPTG) and incubated further under $1 \% \mathrm{CO}_{2}$ until analysis (alongside uninduced controls). The fluorescence was measured at time points 2,4 , and $6 \mathrm{~h}$ after induction using 25 flashes with nine reads per well, normalized to cell density (Tecan infinite 200 PRO), and represented as relative values to allow more convenient comparison between the YFP and GFP datasets.

\section{Ethylene quantitation}

Ethylene production efficiency of the constructed Synechocystis strains was monitored by quantitating ethylene from the headspace of sealed culture vials by GC against a commercial gas standard (AGA; $99 \% \mathrm{~N}_{2}, 1 \% \mathrm{C}_{2} \mathrm{H}_{2}$ v/v [20]. In each case, three biological replicates were sampled at $4 \mathrm{~h}$ after induction using GC-FID (Perkin Elmer AutoSystem) with CP-CarboBOND fused silica capillary column (Varian, $50 \mathrm{~m} \times 0.53 \mathrm{~mm}$ ) under isothermal conditions (oven and injector $80^{\circ} \mathrm{C}$, and detector $200{ }^{\circ} \mathrm{C}$ with

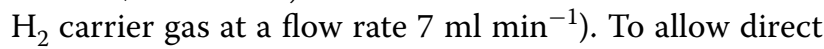
comparison between the samples, ethylene productivity was normalized against culture optical density $(750 \mathrm{~nm}$ ) for calculating average values and corresponding standard deviations.

\section{Statistical comparison of the datasets}

Statistical correlation between the different datasets was evaluated by using two alternative approaches, Pearson correlation coefficient [30] and Spearman's rank correlation [31,32]. The correlation coefficients and the corresponding $\mathrm{p}$ values were calculated in each case from the averaged data using XLSTAT plugin in Microsoft Excel.

\section{Sequence analysis}

Nucleotide sequence analysis of the alternative RBSs in context with the YFP and GFP was performed using the Salis RBS calculator [12] and the UTR Designer [33] for the prediction of the translation initiation rate, using the -25 to +35 sequences of the mRNA transcripts as input (reverse engineering). The same sequences, as well as the entire coding regions ( -25 until the end-codon), were also analyzed using RNAfold Server hosted by ViennaRNA web service [34-36] and mfold web server [37] to predict the most stable mRNA secondary structures with the minimum free energy (MFE).

\section{Additional files}

Additional file 1. Nucleotide sequence of the synthetic GFPmut3b gene codon optimized for Synechosystis sp. PCC 6803.

Additional file 2. List of the expression plasmid constructs generated in this study for evaluating the performance of 13 selected RBS in Synechocystis sp. PCC 6803, using sYFP2, GFPmut3 and efe as quantitative reporters.

Additional file 3. Colony PCR verification of the Synechocystis sp. PCC 6803 strains generated in this study as visualized by agarose gel electrophoresis.

Additional file 4. The full fluorescence activity profiles of the generated Synechocystis sp. PCC 6803 strains expressing sYFP2 and GFPmut3b under the control of the 13 selected RBSs (0-6 h).

Additional file 5. Growth of the Synechocystis sp. PCC 6803 strains expressing SYFP2 under the control of the 13 selected RBS in comparison to the WT control strain (OD $750 \mathrm{~nm}$ at time-points 0,6 and $24 \mathrm{~h}$ ).

Additional file 6. mRNA secondary structure minimum free energy values calculated using RnaFold server and mfold web server prediction tool for the 13 different RBS sequences in context with sYFP2 and GFPmut3b.

Additional file 7. Expression levels predicted by UTR Designer for sYFP2 and GFPmut3b in combination with the 13 target RBS sequences used in the study.

\section{Abbreviations}

Bp: base pairs; Cm: chloramphenicol; D: day; E. coli: Escherichia coli; Em: emission; Ex: exitation; GC: gas chromatography; GFP: GFPmut3b; IPTG: isopropyl- $\beta$ D-1-thiogalactopyranoside; LB: Luria-Bertani; MFE: minimum free energy; OD: optical density; ORF: open reading frame; RBS: ribosome binding site; Rpm: revolutions per minute; SD: Shine-Dalgarno; Sp: spectinomycin; Synechocystis: Synechocystis sp. PCC 6803; WT: wild type; YFP: sYFP2.

\section{Authors' contributions}

KT, EM: experimental design and execution of experimental work. Data analysis, result interpretation and preparation of the manuscript. HD: experimental design, statistical data analysis, result interpretation and preparation of the manuscript. CN: experimental design and preparation of the manuscript. E-MA: support in the conceptual design, data evaluation and manuscript review process. PK: conceptual design and supervision of the research. Data analysis, result interpretation and preparation of the manuscript. All authors read and approved the final manuscript.

\section{Acknowledgements}

We want to acknowledge Professor Ron Milo (Weizmann Institute of Science, IL) for kindly providing the assembly platform pNiv plasmids [3], and Professor Ion Petre (Åbo Akademi University, FI) for the discussions regarding the statistical data analysis.

\section{Competing interests}

The authors declare that they have no competing interests.

\section{Availability of data and supporting materials}

All the data generated and analyzed in this study are included within the article (and its additional files). The research material described in the article is available on request

\section{Consent for publication}

Not applicable.

Ethics approval and consent to participate

Not applicable. 


\section{Funding}

This work was supported by the Tekes-Finnish Funding Agency for Innovation (Grant \#40128/2014); the Academy of Finland (Grants \#271832, \#272424); Finnish Academy of Science and Letters to [H.D.]; Kone Foundation to [H.D.]; and NordForsk NCoE (NordAqua) \#82845.

\section{Publisher's Note}

Springer Nature remains neutral with regard to jurisdictional claims in published maps and institutional affiliations.

\section{Received: 27 December 2017 Accepted: 23 February 2018}

\section{Published online: 02 March 2018}

\section{References}

1. Savakis $\mathrm{P}$, Hellingwerf KJ. Engineering cyanobacteria for direct biofuel production from $\mathrm{CO}_{2}$. Curr Opin Biotechnol. 2015;33:8-14.

2. Lau N-S, Matsui M, Abdullah AA-A. Cyanobacteria: photoautotrophic microbial factories for the sustainable synthesis of industrial products. Biomed Res Int. 2015;2015:754934-43.

3. Zelcbuch L, Antonovsky N, Bar-Even A, Levin-Karp A, Barenholz U, Dayagi M, Liebermeister W, Flamholz A, Noor E, Amram S, et al. Spanning highdimensional expression space using ribosome-binding site combinatorics. Nucleic Acids Res. 2013;41:e98.

4. Heidorn T, Camsund D, Huang H-H, Lindberg P, Oliveira P, Stensjö K, Lindblad P. Synthetic biology in cyanobacteria engineering and analyzing novel functions. Methods Enzymol. 2011;497:539-79.

5. Taton A, Unglaub F, Wright NE, Zeng WY, Paz-Yepes J, Brahamsha B, Palenik B, Peterson TC, Haerizadeh F, Golden SS, et al. Broad-host-range vector system for synthetic biology and biotechnology in cyanobacteria. Nucleic Acids Res. 2014;42:e136.

6. Englund E, Liang F, Lindberg P. Evaluation of promoters and ribosome binding sites for biotechnological applications in the unicellular cyanobacterium Synechocystis sp. PCC 6803. Sci Rep. 2016:6:36640.

7. Xiong W, Morgan JA, Ungerer J, Wang B, Maness PC, Yu J. The plasticity of cyanobacterial metabolism supports direct CO2 conversion to ethylene. Nat Plants. 2015;1:15053.

8. Ramey CJ, Barón-Sola Á, Aucoin HR, Boyle NR. Genome engineering in cyanobacteria: where we are and where we need to go. ACS Synth Biol. 2015:4:1186-96

9. Cardinale S, Arkin AP. Contextualizing context for synthetic biologyidentifying causes of failure of synthetic biological systems. Biotechnol J. 2012;7:856-66.

10. Huang HH, Camsund D, Lindblad P, Heidorn T. Design and characterization of molecular tools for a synthetic biology approach towards developing cyanobacterial biotechnology. Nucleic Acids Res. 2010;38:2577-93.

11. Ma J, Campbell A, Karlin S. Correlations between Shine-Dalgarno sequences and gene features such as predicted expression levels and operon structures. J Bacteriol. 2002;184:5733-45.

12. Salis HM, Mirsky EA, Voigt CA. Automated design of synthetic ribosome binding sites to control protein expression. Nat Biotechnol. 2009;27:946-50.

13. Na D, Lee D. RBSDesigner: software for designing synthetic ribosome binding sites that yields a desired level of protein expression. Bioinformatics. 2010;26:2633-4.

14. Seo SW, Yang JS, Cho HS, Yang J, Kim SC, Park JM, Kim S, Jung GY. Predictive combinatorial design of mRNA translation initiation regions for systematic optimization of gene expression levels. Sci Rep. 2014;4:4515.

15. Markley AL, Begemann MB, Clarke RE, Gordon GC, Pfleger BF. Synthetic biology toolbox for controlling gene expression in the cyanobacterium Synechococcus sp. strain PCC 7002. ACS Synth Biol. 2015;4:595-603.
16. Oliver JWK, Machado IMP, Yoneda H, Atsumi S. Combinatorial optimization of cyanobacterial 2,3-butanediol production. Metab Eng. 2014:22:76-82

17. Wang B, Wang J, Zhang W, Meldrum DR. Application of synthetic biology in cyanobacteria and algae. Front Microbiol. 2012;3:344.

18. Nagai T, Ibata K, Park ES, Kubota M, Mikoshiba K, Miyawaki A. A variant of yellow fluorescent protein with fast and efficient maturation for cellbiological applications. Nat Biotechnol. 2002;20:87-90.

19. Cormack BP, Valdivia RH, Falkow S. FACS-optimized mutants of the green fluorescent protein (GFP). Gene. 1996;173:33-8.

20. Guerrero F, Carbonell V, Cossu M, Correddu D, Jones PR. Ethylene synthesis and regulated expression of recombinant protein in Synechocystis sp. PCC 6803. PLOS ONE. 2012;7:e50470.

21. Nagahama K, Ogawa T, Fujii T, Tazaki M, Tanase S, Morino Y, Fukuda H. Purification and properties of an ethylene-forming enzyme from Pseudomonas syringae pv. phaseolicola PK2. J Gen Microbiol. 1991;137:2281-6.

22. Chartier O, Baker P, Oberč B, Pia, de Jong H, Yagafarova A, Styring P, Bye J, Janssen R, Raschka A, et al. Artificial photosynthesis: potential and reality. European commission, directorate-general for research \& innovation; 2016.

23. Zhou J, Zhang H, Meng H, Zhu Y, Bao G, Zhang Y, Li Y, Ma Y. Discovery of a super-strong promoter enables efficient production of heterologous proteins in cyanobacteria. Sci Rep. 2014;4:4500

24. Reeve B, Hargest T, Gilbert C, Ellis T. Predicting translation initiation rates for designing synthetic biology. Front Bioeng Biotechnol. 2014;2:1.

25. Huang HH, Lindblad P. Wide-dynamic-range promoters engineered for cyanobacteria. J Biol Eng. 2013;7:10.

26. Levin-Karp A, Barenholz U, Bareia T, Dayagi M, Zelcbuch L, Antonovsky N, Noor E, Milo R. Quantifying translational coupling in E. coli synthetic operons using RBS modulation and fluorescent reporters. ACS Synth Biol. 2013;2:327-36.

27. Williams JG. [85] Construction of specific mutations in photosystem II photosynthetic reaction center by genetic engineering methods in Synechocystis 6803. Methods Enzymol. 1988;167:766-78.

28. Rippka R, Deruelles J, Waterbury JB, Herdman M, Stanier RY. Generic assignments, strain histories and properties of pure cultures of cyanobacteria. J Gen Microbiol. 1979;111(1):1-61.

29. Eaton-Rye JJ. Construction of gene interruptions and gene deletions in the cyanobacterium Synechocystis sp. strain PCC 6803. Methods Mol Biol. 2011;684:295-312.

30. Pearson K. III. Contributions to the mathematical theory of evolution. Philos Trans R Soc Lond A. 1894;185:71.

31. Spearman C. The proof and measurement of association between two things. By C. Spearman, 1904. Am J Psychol. 1987;100:441-71.

32. Spearman C. The proof and measurement of association between two things. Int J Epidemiol. 2010;39:1137-50.

33. Seo SW, Yang JS, Kim I, Yang J, Min BE, Kim S, Jung GY. Predictive design of mRNA translation initiation region to control prokaryotic translation efficiency. Metab Eng. 2013:15:67-74.

34. Smith C, Heyne S, Richter AS, Will S, Backofen R. Freiburg RNA Tools: a web server integrating INTARNA, EXPARNA and LOCARNA. Nucleic Acids Res. 2010;38:W373-7

35. Will S, Joshi T, Hofacker IL, Stadler PF, Backofen R. LocARNA-P: accurate boundary prediction and improved detection of structural RNAs. RNA. 2012:18:900-14.

36. Will S, Reiche K, Hofacker IL, Stadler PF, Backofen R. Inferring noncoding RNA families and classes by means of genome-scale structure-based clustering. PLoS Comput Biol. 2007:3:e65.

37. Zuker M. Mfold web server for nucleic acid folding and hybridization prediction. Nucleic Acids Res. 2003:31:3406-15. 